\title{
Actividades deportivas y laborales, y factores asociados a rotura del menisco medial en sujetos no deportistas
}

\author{
Work, sport activities and factors associated with medial meniscal tears in nonathletic \\ subjects
}

\author{
Sergio Charles-Lozoya ${ }^{1 *}$, Joaquín D. Treviño-Báez², Jesús M. Brizuela-Ventura1, \\ Jesús M. Rangel-Flores ${ }^{1}$, Juan C. Tamez-Montes ${ }^{1}$ y Adrián García-Hernández ${ }^{1}$ \\ 'División de Educación en Salud, Servicio de Artroscopia de Rodilla, Unidad Médica de Alta Especialidad, Hospital de Traumatología y Ortopedia \\ No. 21; '2Dirección de Educación e Investigación en Salud, Unidad Médica de Alta Especialidad No. 23. Instituto Mexicano del Seguro Social, \\ Monterrey, Nuevo León, México
}

\section{Resumen}

Antecedentes: Se sabe que la reconstrucción tardía del ligamento cruzado anterior (LCA) se asocia a rotura del menisco medial (MM). Sin embargo, la asociación entre factores referentes a las actividades deportivas, laborales e inestabilidad articular no se ha examinado en sujetos no deportistas. Objetivo: Comparar las actividades laborales, deportivas y otros factores asociados a rotura del MM en sujetos con rotura del LCA. Método: Diseño de casos y controles, de sujetos con rotura del LCA; 140 casos y 140 controles con y sin rotura de MM, respectivamente. Se compararon factores sociodemográficos, actividades deportivas y laborales. Resultados: Los factores independientes asociados a roturas del MM fueron el continuar con actividades deportivas después de la lesión (razón de momios [RM]: 3.6; intervalo de confianza del 95\% [IC 95\%]: 1.7-7.9), la inestabilidad articular (RM: 2.2; IC 95\%: 1.8-2.6), el tiempo de evolución entre la lesión y la reconstrucción (RM: 1.003; IC 95\%: 1.0-1.01) y la edad (RM: 1.1; IC 95\%: 1.03-1.1). Conclusiones: Actividades intensas de la vida diaria, como continuar con deportes, después de una lesión del LCA en sujetos obreros, no deportistas, sin entrenamiento previo, así como la edad, el retraso en la reconstrucción y la inestabilidad articular, son factores de riesgo para rotura del MM.

Palabras Clave: Artroscopia. Factores de riesgo. Ligamento cruzado anterior. Meniscos tibiales. Rodilla.

\begin{abstract}
Background: It is known that late surgical reconstruction of the anterior cruciate ligament (ACL) is associated with a medial meniscal (MM) tears. However, the association between factors relating to sports and work activities and joint instability, has not been examined in non-athletic subjects. Objective: To compare sports and work activities and other factors associated with MM tears, in subjects with ACL rupture. Method: A case-control design study, of patients with ACL injury, 140 cases and 140 controls with and without a rupture of MM respectively, were included. Sociodemographic factors, sports and work activities were compared. Results: The independent factors associated with MM ruptures were continuing sports activities after injury (odds ratio [OR]: 3.6; 95\% confidence interval [95\% Cl]: 1.7-7.9), joint instability (OR: 2.2; 95\% Cl: 1.8-2.6), time between injury and surgical interven-
\end{abstract}

\footnotetext{
Correspondencia:

*Sergio Charles-Lozoya

Avda. José M. Pino Suárez

y Juan Ignacio Ramón, Zona Centro

C.P. 64000 , Monterrey, N.L., México

Fecha de recepción: 21-12-2018

E-mail: scharleslozoya @yahoo.com.mx; $\quad$ Fecha de aceptación: 16-04-2019

sergio.charlesı@imss.gob.mx

DOI: $10.24875 / C I R U .19000986$

0009-7411/๑ 2019 Academia Mexicana de Cirugía. Publicado por Permanyer. Éste es un artículo open access bajo la licencia CC BY-NC-ND (http://creativecommons.org/licenses/by-nc-nd/4.0/).
} 
tion (time of evolution) (OR: 1.003; 95\% Cl: 1.0-1.01) and age (OR: 1.1; 95\% Cl: 1.03-1.1). Conclusions: Intense activities of daily life such as continuing sports activities, after an ACL injury in non-athletic factory workers subjects, without previous training, as well as, age, joint instability and surgical delay are risk factors for rupture of MM.

Key Words: Arthroscopy. Risk factors. Anterior cruciate ligament. Menisci tibial. Knee.

\section{Introducción}

La rotura del ligamento cruzado anterior (LCA) es una lesión común que se asocia con rotura del menisco medial (MM)1. El MM es un estabilizador secundario que previene la traslación anterior de la rodilla cuando está deficiente el LCA. Ante esta deficiencia, el MM se rompe con el paso del tiempo, al sustituir la función estabilizadora del $\mathrm{LCA}^{2}$. Así, se recomienda realizar la reconstrucción antes del año de ocurrida la rotura del $\mathrm{LCA}^{3}$, ya que el retraso en su reconstrucción se asocia a rotura del MM. Sin embargo, esta asociación con otros factores como los de las actividades intensas de la vida diaria en sujetos no deportistas que laboran como operarios, que someten la rodilla a inestabilidad articular constante, tanto en el trabajo como al hacer deporte, no se ha examinado en profundidad, pero podrían interactuar con el tiempo de espera entre la lesión del LCA hasta su reconstrucción y causar lesiones crónicas en la rodilla, provocando incapacidad para laborar. Dentro de los factores de riesgo, la edad se asocia con rotura de MM cuando hay retrasos en el tratamiento ${ }^{4}$. Se refiere que los sujetos mayores de 35 años con rotura del LCA presentan más dolor y discapacidad ${ }^{5}$, además de aparición de cambios degenerativos rápidos en la rodilla, cuando el tiempo de espera para su reconstrucción es mayor de 12 meses $^{6}$. Así mismo, el sexo masculino es un factor que predispone a rotura del $\mathrm{MM}^{7}$. De la misma manera, el número de episodios de inestabilidad articular acumulados antes de la reconstrucción del LCA es un factor asociado a rotura del $\mathrm{MM}^{8}$. Así, se reporta que la edad, el sexo masculino, la reconstrucción tardía mayor de 12 meses y el índice de masa corporal (IMC) son factores de riesgo para rotura del $\mathrm{MM}^{9}$. En cuanto a las actividades laborales en conjunto con las deportivas, se ha reportado, en sujetos sin lesión del LCA, que usar escaleras más de 30 veces al día, acuclillarse en la jornada labora ${ }^{10}$, la obesidad y deportes como él futbol, son factores de riesgo para rotura del $\mathrm{MM}^{11}$, dejando evidencia inconclusa si son los factores laborales, deportivos o ambos los que se asocian a rotura del MM. Así mismo, falta identificar si estas actividades que son parte de la vida activa de un trabajador $^{12}$, en conjunto con la inestabilidad articular constante, el sexo ${ }^{13}$, el IMC y la prolongación del tiempo entre la lesión del LCA y su reconstrucción, predisponen a rotura del MM. Por lo tanto, el objetivo principal de este estudio fue determinar si existe asociación de las actividades laborales, deportivas y otros posibles factores de riesgo con rotura del MM, en sujetos no deportistas, con rotura del LCA.

\section{Método}

\section{Población estudiada y diseño}

Estudio de casos y controles, conducido en el servicio de artroscopia de rodilla del Hospital de Traumatología y Ortopedia No. 21 del Instituto Mexicano del Seguro Social en Monterrey, Nuevo León, México. Durante el periodo de 2016 a 2017, mediante muestreo probabilístico aleatorio simple, se incluyeron pacientes con rotura del LCA, mayores de 18 años, sometidos a reconstrucción artroscópica del LCA, con registro quirúrgico completo y no deportistas. Fueron excluidos los sujetos con cirugía previa, lesiones previas, lesión aguda de MM (diagnosticada por clínica o gabinete), fractura-avulsión del LCA y deformidades de la rodilla. Se calculó el tamaño de la muestra con ayuda del programa Epi Dat 4.1. Se consideró como factor de exposición el retraso en la reconstrucción mayor de 12 meses desde que ocurrió la lesión del LCA hasta su reconstrucción. Para una frecuencia esperada en los casos de un $68 \% \%^{5,12,14,15}$, una razón de momios [RM] a detectar de 2.0 y una relación 1:1, 140 participantes por grupo. Previo a la realización del estudio se contó con la autorización, con número de registro R-2016-1903-15, del Comité Local de Investigación y Ética en Investigación del hospital, y todos los participantes firmaron el consentimiento informado.

\section{Conformación de los grupos}

Los casos y los controles se obtuvieron de una lista de pacientes que fueron operados mediante cirugía de reconstrucción por artroscopia. El grupo de casos 

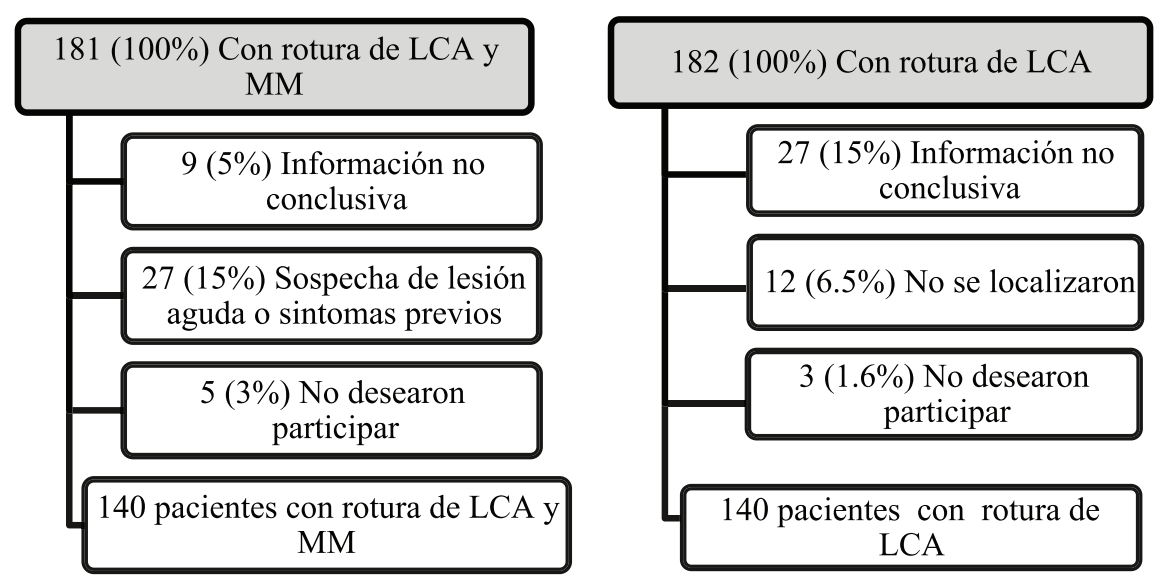

Figura 1. Diagrama de flujo del estudio. LCA: ligamento cruzado anterior; MM: menisco medial.

fueron aquellos que se sometieron a reconstrucción y tuvieron hallazgo de rotura de MM, y el grupo de controles fueron pacientes con rotura del LCA que se sometieron a reconstrucción, sin hallazgo de rotura de MM; así se confirmó la rotura del MM y del LCA. Por lo tanto, la rotura del LCA, y la presencia o no de rotura del MM, debieron haber sido registrados, documentados y dibujados en forma adecuada después de haber sido operados.

\section{Evaluación}

La información fue evaluada a partir de los informes quirúrgicos en forma ciega e independiente por tres médicos residentes (con entrenamiento previo) ajenos a la investigación. El diagnóstico prequirúrgico se hizo por médicos certificados (con adiestramiento en cirugía artroscópica) por quienes realizaron la exploración física y evaluaron los estudios de imagen de aquellos pacientes que los tenían. Para contrastar esta información se analizó la concordancia entre el diagnóstico preoperatorio y los hallazgos reportados en los registros quirúrgicos, obteniéndose una consistencia para un índice kappa de Cohen de 0.81 . Para la recolección de la información de los pacientes se les interrogó directamente en las semanas posteriores a haber sido operados; se expuso el objetivo del estudio y se aplicó un cuestionario específicamente diseñado. Los datos que se investigaron fueron sexo, edad, actividad laboral, actividad deportiva, tipo de deporte, mecanismo de lesión, tiempo de latencia desde la lesión hasta la cirugía de reconstrucción y episodios de inestabilidad (definida como sensación de movilidad anormal por subluxación de la rodilla). Se les preguntó también si trabajaban de pie, si trabajaban sentados, si entrenaban para practicar deportes y si continuaron con actividades deportivas después de la lesión. El tiempo de evolución o latencia desde la lesión se obtuvo a partir de la entrevista con el paciente. Se consideró como un incidente de lesión cuando existió una torsión de la rodilla con un sonido «pop» o por traumatismo directo, ambos acompañados de derrame articular o edema de la rodilla con incapacidad funcional. Se registraron el peso y la talla con ropa ligera, sin zapatos, en una báscula analógica con verificación de calibración y en un estadímetro, en dos ocasiones, y se tomó la media como la medición definitiva, para después calcular el IMC $\left(\mathrm{kg} / \mathrm{m}^{2}\right)$.

\section{Análisis estadístico}

Para el análisis comparativo de variables cuantitativas se usaron las pruebas $t$ de Student $o \mathrm{U}$ de Mann-Whitney de acuerdo con los supuestos de normalidad y homocedasticidad. La prueba de ji al cuadrado de Pearson se utilizó para la comparación de frecuencias y porcentajes. Para evaluar la magnitud de la asociación se midió la RM, con su intervalo de confianza del 95\% (IC 95\%). Se realizó un análisis multivariado mediante el modelo de regresión logística para discriminar aquellos factores que en el análisis bivariado fueron significativos. El valor de significancia fue de alfa $<0.05$. El análisis estadístico se realizó con el programa informático SPSS versión 22 (SPSS, Chicago, IL, EE.UU.).

\section{Resultados}

De los potenciales pacientes para ingresar al estudio fueron elegibles 363, pero se ingresaron 280 (Fig. 1); de ellos, 247 (88.2\%) fueron hombres. La mediana de 
Tabla 1. Características generales de los 280 pacientes con y sin rotura del menisco medial (con rotura del ligamento cruzado anterior)

\begin{tabular}{lccc}
\hline Característica & Con rotura del menisco medial $(\mathbf{n}=140)$ & Sin rotura del menisco medial $(\mathbf{n}=140)$ & $\mathbf{p}$ \\
\hline Edad (años) & $32(25-39)^{\star}$ & $28(23-31)^{\star}$ & $<0.001^{\dagger}$ \\
Sexo masculino & $127(91 \%)$ & $120(86 \%)$ & $0.19^{\ddagger}$ \\
Tabaquismo & $46(33 \%)$ & $43(31 \%)$ & $0.70^{\ddagger}$ \\
Peso $(\mathrm{kg})$ & $84(75-94)^{\star}$ & $78(70-90)^{\star}$ & $0.02^{\dagger}$ \\
Índice de masa corporal $\left(\mathrm{kg} / \mathrm{m}^{2}\right)$ & $28.8(26.5-31.2)^{\star}$ & $27.4(24.9-30)^{\star}$ & $0.004^{\dagger}$ \\
Talla $(\mathrm{m})$ & $1.7(1.65-1.75)^{\star}$ & $1.7(1.65-1.75)^{\star}$ & $0.77^{\dagger}$ \\
Tiempo de latencia & $175(116-414)^{\star}$ & $141(115-181)^{\star}$ & $<0.001^{\dagger}$ \\
Tipo de actividad deportiva & & & $<0.001^{\ddagger}$ \\
Sin actividad deportiva & $53(38 \%)$ & $97(69 \%)$ & $3(2 \%)$ \\
Otros deportes & $9(6 \%)$ & $40(29 \%)$ & \\
Futbol & $78(56 \%)$ & & \\
\hline
\end{tabular}

*Mediana y amplitud intercuartil.

+U de Mann-Whitney.

$\neq \mathrm{Ji}$ al cuadrado.

Tabla 2. Actividades y eventos deportivos en los 280 pacientes con y sin rotura de menisco medial (con rotura del ligamento cruzado anterior)

\begin{tabular}{|c|c|c|c|c|c|}
\hline Actividad & $\begin{array}{l}\text { Con rotura de menisco medial } \\
\qquad(n=140)\end{array}$ & $\begin{array}{l}\text { Sin rotura de menisco medial } \\
\qquad(n=140)\end{array}$ & RM & IC 95\% & $\mathrm{p}$ \\
\hline Episodios de inestabilidad por semana & $3(0-4)^{\star}$ & $0(0-2)^{*}$ & - & - & $<0.001^{\dagger}$ \\
\hline Mecanismo de Lesión indirecto & $121(86 \%)$ & $113(81 \%)$ & 1.5 & $0.8-2.9$ & $0.20^{\ddagger}$ \\
\hline Continuar con Deportes & $87(62 \%)$ & $43(31 \%)$ & 3.7 & $2.2-6.1$ & $<0.001^{\ddagger}$ \\
\hline Entrenamiento deportivo & $57(41 \%)$ & $31(22 \%)$ & 1.4 & $1.4-4.1$ & $0.001^{\ddagger}$ \\
\hline Horas de deporte & $3(0-4)^{\star}$ & $0(0-2)^{*}$ & - & - & $<0.001^{\dagger}$ \\
\hline
\end{tabular}

IC 95\%: intervalo de confianza del 95\%; RM: razón de momios.

*Mediana y amplitud intercuartil.

+U de Mann-Whitney.

‡Ji al cuadrado.

la edad fue de 29 años, con una amplitud intercuartil de 25-34. En cuanto a la profesión, 143 (55\%) eran obreros. Respecto a las actividades deportivas, 118 (43.1\%) practicaban futbol soccer, 150 no hacían actividades deportivas $(53.6 \%), 12$ practicaban otros deportes y $204(72.9 \%)$ hacían deporte sin entrenamiento previo. En cuanto a los posibles factores de riesgo laborales, $67(23.9 \%)$ tenían un trabajo sedentario o predominantemente sentado, $209(74.6 \%)$ trabajaban la mayor parte del tiempo de pie, $203(72.5 \%)$ cargaban peso en su trabajo y $30(10.7 \%)$ usaban escaleras en forma continua en su trabajo. En el grupo de casos hubo diferencias significativas en cuanto a la comparación del tiempo de latencia desde la lesión del LCA hasta la cirugía de reconstrucción, con una diferencia de 34 días respecto al grupo control $(p<0.001)$; el tiempo de evolución desde iniciada la lesión en la población total tuvo una mediana de 152.5 días, con una amplitud intercuartil de 115.5-370.75. Al analizar otros posibles factores de riesgo asociados con la rotura del MM en los casos, se observó que estos eran de mayor edad, de mayor peso y de mayor IMC (Tabla 1). Así mismo, se compararon otros posibles factores asociados con la rotura del MM, como los episodios de inestabilidad, el entrenamiento deportivo, el tiempo en horas dedicadas a las actividades deportivas y el continuar con actividades deportivas después de la lesión, mostrando diferencias estadísticas significativas (Tabla 2). En los factores de riesgo laboral se observó que trabajar sentado fue un probable factor protector, así como que trabajar de pie y cargar peso fueron posibles factores de riesgo (Tabla 3). En el análisis multivariado mediante el modelo de regresión logística, la edad, el tiempo de latencia prolongado, los episodios de inestabilidad y el continuar con actividades deportivas fueron significativos (Tabla 4). 
Tabla 3. Características de las actividades laborales en los 280 pacientes con y sin rotura de menisco medial (con rotura del ligamento cruzado anterior)

\begin{tabular}{lccccc}
\hline Actividad & Con rotura de menisco medial $(\mathbf{n = 1 4 0 )}$ & Sin rotura de menisco medial $(\mathbf{n}=\mathbf{1 4 0})$ & $\mathbf{R M}$ & $\mathbf{I C} \mathbf{9 5 \%}$ & $\mathbf{p}$ \\
\hline Maneja en trabajo & $6(4 \%)$ & $11(8 \%)$ & 0.5 & $0.2-1.5$ & $0.21^{*}$ \\
Trabaja sentado & $24(17 \%)$ & $43(31 \%)$ & 0.5 & $0.3-0.8$ & $0.008^{*}$ \\
Trabaja de pie & $113(81 \%)$ & $96(69 \%)$ & 1.9 & $1.1-3.3$ & $0.02^{*}$ \\
Carga peso & $111(79 \%)$ & $92(66 \%)$ & 2.0 & $1.2-3.4$ & $0.01^{*}$ \\
Usa escaleras & $18(13 \%)$ & $12(9 \%)$ & 1.6 & $0.73-3.4$ & $0.25^{*}$ \\
\hline
\end{tabular}

IC 95\%: intervalo de confianza del 95\%; RM: razón de momios.

*Ji al cuadrado.

Tabla 4. Variables en la ecuación de regresión logística para la asociación. Se tomó como referencia para el análisis la presencia o ausencia de lesión del menisco medial

\begin{tabular}{|c|c|c|c|c|c|c|c|}
\hline Paso 4 & B & ES & $\chi^{2}$ Wald & gl & $p$ & Exp B & IC $95 \%$ \\
\hline Edad & 0.080 & 0.026 & 9.524 & 1 & 0.002 & 1.1 & $1.04-1.14$ \\
\hline $\begin{array}{l}\text { Continuar con } \\
\text { actividades } \\
\text { deportivas }\end{array}$ & 1.285 & 0.397 & 10.463 & 1 & 0.001 & 3.6 & $1.7-7.9$ \\
\hline $\begin{array}{l}\text { Tiempo de } \\
\text { latencia }\end{array}$ & 0.003 & 0.001 & 4.533 & 1 & 0.033 & 1.003 & $1.0-1.01$ \\
\hline $\begin{array}{l}\text { Inestabilidad } \\
\text { articular }\end{array}$ & 0.745 & 0.089 & 69.662 & 1 & $<0.001$ & 2.2 & $1.8-2.6$ \\
\hline Constante & -7.680 & 1.052 & 53.320 & 1 & $<0.001$ & 0.000 & \\
\hline
\end{tabular}

B: coeficiente de regresión logistica; $\mathrm{ES}$ : error estándar; Exp B: exponente $\mathrm{B}$ (odds ratio); gl: grados de libertad; IC 95\%: intervalo de confianza del $95 \% ; \chi^{2}$ : ji al cuadrado de Wald.

\section{Discusión}

En este estudio se observó que si el tiempo de latencia desde la lesión hasta la cirugía de reconstrucción del LCA es prolongado se asocia más con la complicación de rotura del $\mathrm{MM}$, al igual que en el estudio de Chhadia, et al. ${ }^{16}$, quienes determinaron un riesgo de rotura del MM dos veces mayor en los sujetos con 12 meses de tiempo de latencia hasta la reconstrucción. Anstey, et al. ${ }^{14}$ refieren que, a 6 meses de ocurrida la lesión, aumenta la prevalencia de rotura del MM del 4.1 a $16.7 \%$. Otros estudios reportan que, a 12 meses de evolución, la incidencia de rotura del MM aumenta al $80 \%{ }^{17}$, y que por cada mes de tiempo de espera entre la lesión y su reconstrucción se incrementa un $1 \%^{18}$ y por cada año un $6 \%{ }^{19}$.

En cuanto a la edad y el tiempo de latencia, Magnussen, et al. ${ }^{20}$ sustentan que los sujetos con rotura de LCA mayores de 22 años con un tiempo de espera de cirugía mayor a 12 semanas presentan rotura del MM con más frecuencia. Así mismo, Tandogan, et al. ${ }^{4}$ observaron que los pacientes con lesiones complejas de menisco son 3 años mayores que aquellos con lesiones simples. En esta investigación, los casos tuvieron una mediana de edad de 32 años (rango: 18-51) y un tiempo de latencia de 175 días; esto puede deberse a la degeneración que se presenta con la edad en el tejido del menisco, que en conjunto con el retraso en la reconstrucción del LCA se asocia a rotura del MM.

Por otra parte, Brambilla, et al. ${ }^{9}$ determinaron que la lesión del LCA más un IMC elevado se asocia con rotura de $\mathrm{MM}$, en coincidencia con este reporte, al observar que los casos con rotura de MM tuvieron un IMC más alto que el de los controles, pero ambos con sobrepeso.

El sexo es otro factor de riesgo y así lo sustentan Kluczynski, et al. ${ }^{13}$, quienes refieren que en el sexo masculino es dos veces más frecuente la rotura de MM. Brambilla, et al. ${ }^{9}$ estimaron datos similares (RM: 1.4-2.6). En este reporte tuvimos una prevalencia alta de hombres $(88.2 \%)$, por lo que no se observaron diferencias, lo que muestra que en nuestra población los hombres presentan más roturas del LCA debido a las actividades deportivas (en nuestro estudio, el $78.8 \%$ de las mujeres no practicaba deportes).

Otro factor de riesgo son los episodios de inestabilidad articular. Kluczynski, et al. ${ }^{8}$ observaron que más de cuatro episodios de inestabilidad, sin especificar la intensidad ni el tiempo, se asocian más a rotura de MM. Nosotros obtuvimos una mediana de tres episodios a la semana, por lo que es probable que sea más frecuente de lo que se ha descrito. De igual manera, Joseph, et al. ${ }^{21}$ sustentan que los sujetos que no hacen actividades deportivas y sufren retraso en la reconstrucción del LCA son igual de susceptibles a sufrir una lesión del MM, por lo que surge la hipótesis de si la inestabilidad articular por sí misma podría predisponer a lesión del MM. Por ello, deberían hacerse estudios comparativos con el uso de dispositivos ortopédicos que eviten la traslación anormal de 
la articulación y así poder evaluar la influencia de otros factores de riesgo.

En cuanto a la actividad deportiva, Tandogan, et al. ${ }^{4}$ sustentan que la práctica del futbol soccer se asocia a rotura del MM en un $65 \%$ de los sujetos de su reporte, pero no encuentran asociación con el nivel de entrenamiento previo, en concordancia con este análisis, en el que la rotura de MM fue más frecuente en los sujetos que practicaban futbol soccer. Sin embargo, en cuanto al nivel de entrenamiento, nosotros observamos que las horas totales de práctica deportiva por semana, el entrenamiento deportivo y seguir practicando deportes de manera recreativa después de la lesión fueron factores asociados con rotura del MM $(p=0.001)$. Del mismo modo, Chen, et al..$^{12}$ refieren que los sujetos que continúan con actividades intensas después de la lesión del LCA, incluidos los que practican deportes de alta intensidad con regularidad, en comparación con los inactivos o que hacen deportes de menor intensidad, presentan rotura del MM con más frecuencia. Así mismo, Baker, et al. ${ }^{10}$ analizaron actividades deportivas y laborales, pero en sujetos sin rotura del LCA, y señalan que practicar deportes de alta demanda funcional, como el futbol soccer, encuclillarse o arrodillarse durante el trabajo, $\mathrm{y}$ tener obesidad, se asocian con rotura del MM; igualmente, refieren que los sujetos que se hincan o acuclillan y que suben escaleras más de 30 veces al día durante la jornada laboral tienen mayor asociación con rotura de $\mathrm{MM}^{11}$. Nosotros encontramos que usar escaleras de manera continua durante el trabajo no fue significativo, y no tuvimos sujetos que se encuclillaran o arrodillaran continuamente en el trabajo. Sin embargo, tener sujetos que cargaban peso o estaban de pie durante la jornada laboral fue frecuente y se asoció a rotura del MM. Trabajar sentado fue un posible factor protector para la rotura del MM; en este reporte, el $52.2 \%$ de los sujetos que laboraban sentados no realizaba actividad deportiva. Por tanto, un punto de debate a considerar es si se deben continuar las actividades laborales en este tipo de sujetos o mantenerlos incapacitados, con los costos que ello sobrelleva.

Las limitantes de este estudio incluyen que no se tomaron en cuenta las lesiones del cartílago articular ni del menisco lateral, y que los pacientes al inicio de la lesión no tuvieron un estudio de resonancia magnética que confirmara o descartara la rotura de $\mathrm{MM}$ simultáneamente con la rotura del LCA, lo que pudo causar un sesgo de clasificación. Otro aspecto que no se investigó fue la escolaridad, para evaluar su posible efecto en la búsqueda pertinente de atención médica especializada.

Las fortalezas son que la muestra fue representativa y homogénea, la población estudiada se seleccionó en forma simultánea y se investigaron factores de riesgo que han sido analizados en forma aislada en otras investigaciones.

\section{Conclusiones}

En síntesis, un factor común en el tipo de población estudiada fue que la articulación de la rodilla se sometió a mayor estrés biomecánico debido a las actividades intensas de la vida diaria y a continuar con actividades deportivas después de una rotura del LCA en sujetos no deportistas que son operarios, sin acondicionamiento físico adecuado. Tener mayor edad, sufrir retrasos en la reconstrucción del LCA y la inestabilidad articular constante podrían ser factores de riesgo para rotura del MM. Por lo tanto, se deberían evitar los deportes de alta demanda funcional en este tipo de sujetos, ya que la actividad laboral podría predisponer por sí misma a rotura del MM, y aunque en este reporte no fue un factor independiente asociado, debe tomarse en consideración, por lo que se recomienda realizar más investigaciones al respecto.

\section{Conflicto de intereses}

Los autores declaran no tener ningún conflicto de intereses.

\section{Financiamiento}

Ninguno.

\section{Responsabilidades éticas}

Protección de personas y animales. Los autores declaran que para esta investigación no se han realizado experimentos en seres humanos ni en animales.

Confidencialidad de los datos. Los autores declaran que han seguido los protocolos de su centro de trabajo sobre la publicación de datos de pacientes.

Derecho a la privacidad y consentimiento informado. Los autores han obtenido el consentimiento informado de los pacientes y/o sujetos referidos en el artículo. Este documento obra en poder del autor de correspondencia. 


\section{Bibliografía}

1. Kilcoyne KG, Dickens JF, Haniuk E, Cameron KL, Owens BD. Epidemiology of meniscal injury associated with $\mathrm{ACL}$ tears in young athletes. Orthopedics. 2012;35:208-12.

2. Widener DB, Wilson DJ, Galvin JW, Marchant BG, Arrington ED. The prevalence of meniscal tears in young athletes undergoing revision anterior cruciate ligament reconstruction. Arthroscopy. 2015;31:680-3.

3. Kennedy J, Jackson MP, O'Kelly P, Moran R. Timing of reconstruction of the anterior cruciate ligament in athletes and the incidence of secondary pathology within the knee. J Bone Joint Surg Br. 2010;92: 362-6.

4. Tandogan RN, Taşer O, Kayaalp A, Taşkiran E, Pinar H, Alparslan B, et al. Analysis of meniscal and chondral lesions accompanying anterior cruciate ligament tears: relationship with age, time from injury, and level of sport. Knee Surg Sports Traumatol Arthrosc. 2004;12:262-70.

5. Fok AW, Yau WP. Delay in ACL reconstruction is associated with more severe and painful meniscal and chondral injuries. Knee Surg Sports Traumatol Arthrosc. 2013;21:928-33.

6. Roos H, Adalberth T, Dahlberg L, Lohmander LS. Osteoarthritis of the knee after injury to the anterior cruciate ligament or meniscus: the influence of time and age. Osteoarthritis Cartilage. 1995;3:261-7.

7. Unay K, Akcal MA, Gokcen B, Akan K, Esenkaya I, Poyanlı O. The relationship between intra-articular meniscal, chondral, and ACL lesions: finding from 1,774 knee arthroscopy patients and evaluation by gender. Eur J Orthop Surg Traumatol. 2014;24:1255-62.

8. Kluczynski MA, Marzo JM, Bisson LJ. Factors associated with meniscal tears and chondral lesions in patients undergoing anterior cruciate ligament reconstruction: a prospective study. Am J Sports Med. 2013 41:2759-65.

9. Brambilla L, Pulici L, Carimati G, Quaglia A, Prospero E, Bait C et al. Prevalence of associated lesions in anterior cruciate ligament reconstruction: correlation with surgical timing and with patient age, sex, and body mass index. Am J Sports Med. 2015:43:2966-73.

10. Baker P, Coggon D, Reading I, Barrett D, McLaren M, Cooper C. Sports injury, occupational physical activity, joint laxity, and meniscal damage. J Rheumatol. 2002;29:557-63.
11. Baker P, Reading I, Cooper C, Coggon D. Knee disorders in the general population and their relation to occupation. Occup Environ Med. 2003;60:794-7.

12. Chen G, Tang X, Li Q, Zheng G, Yang T, Li J. The evaluation of patient-specific factors associated with meniscal and chondral injuries accompanying ACL rupture in young adult patients. Knee Surg Sports Traumatol Arthrosc. 2015;23:792-8.

13. Kluczynski MA, Marzo JM, Rauh MA, Bernas GA, Bisson LJ Sex-specific predictors of intra-articular injuries observed during anterior cruciate ligament reconstruction. Orthop J Sports Med. 2015;3:2325967115571300.

14. Anstey DE, Heyworth BE, Price MD, Gill TJ. Effect of timing of ACL reconstruction in surgery and development of meniscal and chondral lesions. Phys Sportsmed. 2012;40:36-40.

15. Mei Y, Ao Y, Wang J, Ma Y, Zhang X, Wang J, et al. Clinical characteristics of 4355 patients with anterior cruciate ligament injury. Chin Med J (Engl). 2013;126:4487-92.

16. Chhadia AM, Inacio MC, Maletis GB, Csintalan RP, Davis BR, Funahashi TT. Are meniscus and cartilage injuries related to time to anterior cruciate ligament reconstruction? Am J Sports Med. 2011;39:1894-9.

17. Ralles S, Agel J, Obermeier M, Tompkins M. Incidence of secondary intra-articular injuries with time to anterior cruciate ligament reconstruction. Am J Sports Med. 2015;43:1373-9.

18. Granan L-P, Bahr R, Lie SA, Engebretsen L. Timing of anterior cruciate ligament reconstructive surgery and risk of cartilage lesions and meniscal tears: a cohort study based on the Norwegian National Knee Ligament Registry. Am J Sports Med. 2009;37:955-61.

19. Michalitsis S, Vlychou M, Malizos KN, Thriskos P, Hantes ME. Meniscal and articular cartilage lesions in the anterior cruciate ligament-deficient knee: correlation between time from injury and knee scores. Knee Surg Sports Traumatol Arthrosc. 2015;23:232-9.

20. Magnussen RA, Pedroza AD, Donaldson CT, Flanigan DC, Kaeding CC. Time from ACL injury to reconstruction and the prevalence of additional intra-articular pathology: is patient age an important factor? Knee Surg Sports Traumatol Arthrosc. 2013;21:2029-34.

21. Joseph C, Pathak SS, Aravinda M, Rajan D. Is ACL reconstruction only for athletes? A study of the incidence of meniscal and cartilage injuries in an ACL-deficient athlete and non-athlete population: an Indian experience. Int Orthop. 2008;32:57-61. 